\title{
Identification of a Novel Human
}

Papillomavirus, Type HPV199, Isolated from

a Nasopharynx and Anal Canal, and Complete Genomic Characterization of Papillomavirus Species Gamma-12

\author{
Anja Oštrbenk ${ }^{1}$, Boštjan J. Kocjan ${ }^{1}$, Lea Hošnjak ${ }^{1}$, Jingjing Li ${ }^{2}$, Qiuju Deng ${ }^{2}$, \\ Anja Šterbenc ${ }^{1}$, Mario Poljak ${ }^{1 *}$ \\ 1 Institute of Microbiology and Immunology, Faculty of Medicine, University of Ljubljana, Ljubljana, Slovenia, \\ 2 Key Laboratory of Carcinogenesis and Translational Research (Ministry of Education), Peking University \\ Cancer Hospital \& Institute, Beijing, China \\ *mario.poljak@mf.uni-lj.si
}

\section{f open ACCESS}

Citation: Oštrbenk A, Kocjan BJ, Hošnjak L, Li J, Deng Q, Šterbenc A, et al. (2015) Identification of a Novel Human Papillomavirus, Type HPV199, Isolated from a Nasopharynx and Anal Canal, and Complete Genomic Characterization of Papillomavirus Species Gamma-12. PLOS ONE 10(9): e0138628. doi:10.1371/journal.pone. 0138628

Editor: Peter C. Angeletti, University of NebraskaLincoln, UNITED STATES

Received: June 3, 2015

Accepted: August 31, 2015

Published: September 16, 2015

Copyright: @ 2015 Oštrbenk et al. This is an open access article distributed under the terms of the Creative Commons Attribution License, which permits unrestricted use, distribution, and reproduction in any medium, provided the original author and source are credited.

Data Availability Statement: All relevant data are within the paper and its Supporting Information files.

Funding: This work is the result of $\mathrm{PhD}$ research and is financially supported by the Slovenian Research Agency (ARRS), which funded AO through the Young Researcher Training Program, and in part by the CoheaHr Project (European Commission FP7 Program, grant number: 603019) (MP). The funders had no role in study design, data collection and analysis, decision to publish, or preparation of the manuscript.

\section{Abstract}

The novel human papillomavirus type 199 (HPV199) was initially identified in a nasopharyngeal swab sample obtained from a 25 year-old immunocompetent male. The complete genome of HPV199 is 7,184 bp in length with a GC content of $36.5 \%$. Comparative genomic characterization of HPV199 and its closest relatives showed the classical genomic organization of Gammapapillomaviruses (Gamma-PVs). HPV199 has seven major open reading frames (ORFs), encoding five early (E1, E2, E4, E6, and E7) and two late (L1 and L2) proteins, while lacking the E5 ORF. The long control region (LCR) of $513 \mathrm{bp}$ is located between the L1 and E6 ORFs. Phylogenetic analysis additionally confirmed that HPV-199 clusters into the Gamma-PV genus, species Gamma-12, additionally containing HPV127, HV132, HPV148, HPV165, and three putative HPV types: KC5, CG2 and CG3. HPV199 is most closely related to HPV127 (nucleotide identity $77 \%$ ). The complete viral genome sequence of additional HPV199 isolate was determined from anal canal swab sample. Two HPV199 complete viral sequences exhibit $99.4 \%$ nucleotide identity. To the best of our knowledge, this is the first member of Gamma-PV with complete nucleotide sequences determined from two independent clinical samples. To evaluate the tissue tropism of the novel HPV type, 916 clinical samples were tested using HPV199 type-specific real-time PCR: HPV199 was detected in 2/76 tissue samples of histologically confirmed common warts, 2/108 samples of eyebrow hair follicles, 2/137 anal canal swabs obtained from individuals with clinically evident anal pathology, 4/184 nasopharyngeal swabs and 3/411 cervical swabs obtained from women with normal cervical cytology. Although HPV199 was found in $1.4 \%$ of cutaneous and mucosal samples only, it exhibits dual tissue tropism. According to the results of our study and literature data, dual tropism of all Gamma-12 members is highly possible. 
Competing Interests: The authors have declared that no competing interests exist.

\section{Introduction}

Human papillomaviruses (HPVs) are small, non-enveloped DNA viruses with a circular, double stranded genome of approximately 8,000 base pairs (bp). They are a group of highly divergent viruses that infect human epithelial cells, where they are implicated in the development of several benign and malignant neoplasms [1, 2]. HPVs are hierarchically classified into types, species and genera: HPV types that display less than $60 \%$ similarity in the L1 open reading frame (ORF) nucleotide sequence are classified to different genera, while viral species within the same genus share $60-70 \%$ similarity [1]. The taxonomy of papillomaviruses at the species level and above is verified by the International Committee for the Taxonomy of Viruses; however, HPV type designation and classification below the species level is maintained by the International HPV Reference Center at the Karolinska Institute, Stockholm, Sweden [3]. A consecutive number is assigned to a novel HPV type only after the following conditions are met: (i) the putative novel HPV type shows less than $90 \%$ nucleotide sequence identity with all known HPV types and (ii) a reference clone is deposited at the HPV reference center, where they re-clone and re-sequence the putative novel HPV type [1,3]. As of May 30, 2015, two hundred and one different HPV types, ranging from HPV-1 to HPV-205, were officially recognized: 65 Alphapapillomaviruses (Alpha-PVs), 51 Betapapillomaviruses (Beta-PVs), 81 Gammapapillomaviruses (Gamma-PVs), 3 Mupapillomaviruses (Mu-PVs), and a single Nupapillomavirus (Nu-PV) (http://www.hpvcenter.se/html/refclones.html). Four previously recognized HPV types (HPV-46, HPV-55, HPV-64 and HPV-79) were recently re-classified as subtypes.

HPV types have traditionally been classified as mucosal or cutaneous on the assumption that HPV tissue tropism reflects the location from which the original isolate of the particular type was found [4, 5]. Accordingly, HPV types belonging to the Alpha-PVs are predominantly assigned as anogenital types and Beta-PVs, Gamma-PVs, $\mathrm{Mu}$-PVs and $\mathrm{Nu}$-PVs as cutaneous types [2, 4]. However, a growing body of evidence suggests that HPV types belonging to Gamma-PVs (the genus that has been growing most rapidly in recent years and is currently divided into 27 species) are ubiquitous and show much broader tissue tropism than previously thought [5], with reported detection sites ranging from healthy skin and various cutaneous lesions [6-9] to genital lesions [10] and oral [4] and nasal [11] mucosa. One recent report suggests that Gamma-PVs are also able to infect mucocutaneous sites such as the anal canal [12].

In the present study, we report a novel Gamma-PV type, HPV199, originally found in a nasopharyngeal swab sample obtained from a 25 year-old healthy individual. We fully characterized and phylogenetically evaluated the complete genome sequences of HPV199 and its closest relatives. The novel HPV type belongs to species Gamma-12, which so far contains only four officially recognized HPV types: HPV127, HPV132, HPV148 and HPV165 and 3 putative novel HPV types: CG2, CG3 and KC5 [3, 5]. The HPV199 genome organization was further investigated by comparison of the long control region, ORFs and protein functional domains with its closest relatives. The complete viral genome sequence of additional HPV199 isolate was determined from anal canal swab sample. Furthermore, an HPV199 type-specific RT-PCR was developed in order to test a representative collection of various HPV-associated clinical specimens to ascertain the tissue tropism of HPV199.

\section{Materials and Methods}

\section{Identification of a partial HPV199 331 bp L1 gene sequence}

In order to identify novel HPV types, stored DNA samples isolated from nasopharyngeal swabs were tested using different primer sets targeting a broad spectrum of HPV types 
belonging to the Alpha-PV, Beta-PV and Gamma-PV genera, as described previously [13-17]. Swabs were collected from 75 men and 100 women (age range 0-77 years, median age 28.0 years) with suspected whooping cough who had been referred for routine PCR testing for Bordetella pertussis. PCR products of the expected sizes were purified using a QIAquick PCR Purification Kit (Qiagen) and processed for sequence analysis and HPV type determination, as described previously [13]. In a nasopharyngeal swab obtained from a 25 year-old immunocompetent male, a partial HPV L1 gene sequence of $331 \mathrm{bp}$ in length was obtained using the broadrange FAP6085F/FAP64 PCR protocol, as described previously [17], which did not correspond to any of the already established HPV types. This initial HPV199 sequence was deposited in the GenBank sequence database in November 2013, under accession number HG515499.

Total DNA from the original nasopharyngeal sample containing HPV199 was isolated using a QiAamp DNA Investigator Kit (Qiagen, Hilden, Germany), following a slightly modified protocol for isolation of DNA from surface and buccal swabs (Qiagen). Briefly, swabs were placed in a $1.5 \mathrm{ml}$ microcentrifuge tube with $20 \mu \mathrm{l}$ of proteinase $\mathrm{K}$ and $180 \mu \mathrm{l}$ of buffer ATL and incubated for $15 \mathrm{~min}$ at $56^{\circ} \mathrm{C}$. After incubation, $200 \mu \mathrm{l}$ of buffer AL, $1 \mu \mathrm{l}$ of carrier RNA $(1 \mu \mathrm{g} / \mu \mathrm{l})$ and $200 \mu \mathrm{l}$ of ethanol (96-100\%) were added to the samples, mixed thoroughly and incubated for $5 \mathrm{~min}$ at room temperature. Each sample was transferred to a QIAamp MinElute column and centrifuged for $1 \mathrm{~min}$ at $8,000 \mathrm{rpm}$. The columns were washed once with $500 \mu \mathrm{L}$ of AW1-buffer, once with $700 \mu \mathrm{L}$ of AW2-buffer and once with $700 \mu \mathrm{L}$ of ethanol, and centrifuged each time for $1 \mathrm{~min}$ at $8,000 \mathrm{rpm}$. This was followed by centrifugation of a column for 3 $\min$ at $14,000 \mathrm{rpm}$. Bound DNA was eluted with $100 \mu \mathrm{L}$ of ATE buffer and stored at $-20^{\circ} \mathrm{C}$ until molecular analysis.

\section{PCR amplification of a full genome of HPV199}

Based on the HPV199 L1 partial 331 bp sequence, a primer set KC82-LNG-F ( 5 ' - GGCAA TAAGGGTGATTGTCCT-3 ', nt 5,694-5,714) and KC82-LNG-R (5 ' - TTTTTTACAAGGTT CAGCAACATC-3 ', nt 5,693-5,670), for the inverse long-range PCR was manually constructed using the BioEdit software package [18] and evaluated and corrected using the Netprimer program (http://www.premierbiosoft.com/netprimer/index.html). Prior to full-genome amplification of HPV199, sample DNA was subjected to rolling circle amplification (RCA) using an Ilustra TempliPhi ${ }^{\mathrm{TM}} 100$ Amplification Kit (GE Healthcare, Amersham, UK), as described previously [19]. The obtained RCA product was diluted in nuclease free water (ratio 1:100) and used in the subsequent PCR reaction.

A complete viral genome was amplified in several replicates using a Platinum Taq DNA Polymerase High Fidelity Kit (Invitrogen, Carlsbad, USA). The reaction mixture had a final volume of $25 \mu \mathrm{l}$ and contained $5 \mu \mathrm{l}$ of diluted RCA product, $2.5 \mu \mathrm{l}$ of 10X High Fidelity PCR Buffer, $1 \mu \mathrm{l}$ of $50 \mathrm{mM} \mathrm{MgSO}_{4}, 200 \mu \mathrm{M}$ of dNTPs, $0.5 \mathrm{U}$ of Platinum Taq DNA Polymerase High Fidelity, $0.4 \mu \mathrm{M}$ of each primer, and water. PCR-amplification was performed on a Veriti Thermal Cycler (Applied Biosystems, Foster City, USA) under the following conditions: 2 min of denaturation at $94^{\circ} \mathrm{C}$, followed by 45 cycles of $30 \mathrm{~s}$ at $94^{\circ} \mathrm{C}, 30 \mathrm{~s}$ at $54^{\circ} \mathrm{C}$ and $8 \mathrm{~min}$ at $68^{\circ} \mathrm{C}$, and a final elongation step of $7 \mathrm{~min}$ at $68^{\circ} \mathrm{C}$. After amplification was completed, the reaction mixture was cooled to $8^{\circ} \mathrm{C}$.

\section{Sequencing and cloning of the complete HPV199 genome}

After amplification, the obtained PCR products were separated by gel electrophoresis and visible bands of the expected size were purified with a QIAquick PCR Purification Kit (Qiagen). One PCR product was selected to determine the viral full-genome sequence using a primer walking strategy at Peking University Cancer Hospital \& Institute. Primers used for HPV199 
whole genome sequencing are listed in S1 Table. This particular amplicon was additionally cloned into pCR-XL-TOPO plasmid vector using a TOPO XL PCR Cloning Kit (Invitrogen), according to the manufacturer's instructions. After transformation of One Shot TOP10 chemically competent E. coli cells (Invitrogen) with HPV199 plasmids, the transformants were incubated overnight at $37^{\circ} \mathrm{C}$ on LB plates containing $50 \mu \mathrm{g} / \mathrm{mL}$ kanamycin. Bacterial colonies containing a fragment of the appropriate size were selected by colony PCR using FastStart PCR Master (Roche diagnostics) and M13 Forward (-20)/M13 reverse primers (Invitrogen), and grown overnight in LB kanamycin $(50 \mu \mathrm{g} / \mathrm{mL})$ medium. HPV199 plasmid DNA was extracted from $4 \mathrm{ml}$ of bacterial culture using a QIAprep Spin Miniprep Kit (Qiagen), as instructed by the manufacturer. To avoid possible differences in nucleotide sequences of HPV199 PCR amplicons and plasmid clones, primer walking was repeated on one clone using identical primers (S1 Table). HPV199 plasmid DNA was sequenced in in-house sequence facilities using a Big Dye Terminator v3.1 Cycle Sequencing Kit (PE Applied Biosystems, Foster City, CA), as described previously [20], and analyzed on 3500 Series Genetic (PE Applied Biosystems, Foster City, CA).

\section{HPV199 genomic characterization}

The complete nucleotide sequence of the HPV199 genome was assembled and edited with the Vector NTI Advance 11 program package (Invitrogen). ORFs of HPV199 were predicted by the ORF Finder Tool (http://www.ncbi.nlm.nih.gov/gorf/gorf.html) and verified with Vector NTI. The HPV199 genome organization was further investigated by comparing nucleotide alignments of individual genes of HPV199 and its closest relatives using the MEGA6 software package [21]. The detailed characterization of LCR, ORFs and protein functional domains of HPV199 and its closest relatives was done as described previously [22].

\section{Phylogenetic analysis}

The complete genomes from all completely sequenced Gamma-PVs, including all putative novel types pending classification at the HPV reference center, were obtained from the Papillomavirus Episteme database (PaVE) [23] and aligned by the MUSCLE algorithm [24, 25] of the MEGA6 software package [21]. The first ATG of E6 was defined as the starting position for the sequence alignment, with the exception of HPV101 and HPV103, which lack E6 proteins [26]. The nucleotide sequences of two Beta-PVs, HPV5 and HPV8, were used to root the tree. All further phylogenetic analyses were performed with MEGA6. A General Time Reversible (GTR) model with five Gamma discrete categories $(+\mathrm{G})$, with an allowance for the presence of invariant sites (+I), was determined by MEGA6 to be the best fitting nucleotide substitution model. In order to construct the phylogenetic tree, the maximum likelihood algorithm was used, with 1,000 bootstrap replicates. A graphic presentation of the phylogenetic tree was made with FigTree software v1.4.2 (http://tree.bio.ed.ac.uk/software/figtree/).

The phylogenetic relationship of HPV199 with other members of species Gamma-12 was analyzed using the EMBOSS Water Pairwise Sequence Alignment tool (http://www.ebi.ac.uk/ Tools/psa/emboss_water/), which uses the Smith-Waterman algorithm to calculate the local alignment of individual sequences. With this method, we performed pairwise alignment of nucleotide and amino acid sequences for all seven viral proteins and the LCR region and calculated the percentage similarity. The phylogenetic relationship was further confirmed with the PASC tool at NCBI [27], which uses the Needleman-Wunsch algorithm to calculate the global alignment of sequences based on the complete genome. In order to determine whether two genomes exhibit similar degrees of homology across different regions of their genomes, the 
ratios of relatedness (L1:E1 ORF ratio) were calculated for all known and putative HPV types within Gamma-12 [28, 29].

\section{HPV199 type-specific real-time PCR}

HPV199 type-specific primers and a probe for quantitative RT-PCR were designed within the HPV199 L2 gene, using on-line primer/probe design software (http://eu.idtdna.com/ PrimerQuest/Home/Index). The designed primers KC82RTF ( 5 ' - CATTAGAGCGTGTAG CAACTCGT-3 ', nt 4,364-4,386) and KC82RTR (5 ' -TGCTCAAATTGTAATGTGACATCTC$3^{\prime}$, nt 4,499-4,523) resulted in a PCR product of 160 bp in length. A 5' FAM/ZEN-labeled Taqman probe KC82RTSR ( 5 ' -AAGGCGGGAAGGCTGAAG-3 ' , nt 4,447-4,464) was additionally constructed to monitor the specific real-time amplification of the targeted genomic fragment. In order to avoid cross-reactivity with closely related HPV types, the selected primers and probes were analyzed using NCBI Blast and specificity was additionally confirmed by sequencing all HPV199 RT-PCR positive amplicons.

The reaction mixture of the optimized HPV199 RT-PCR assay had a total volume of $20 \mu \mathrm{l}$, containing $5 \mu \mathrm{l}$ of DNA sample, $10 \mu \mathrm{l}$ of $2 \mathrm{X}$ LightCycler 480 Probes Master (Roche Diagnostics), $0.5 \mu \mathrm{M}$ of each primer and probe, and $4.4 \mu \mathrm{l}$ of water. RT-PCR was carried out in a 96-well plate on a LightCycler 480 II RT-PCR Instrument (Roche) under the following cycling conditions: $10 \mathrm{~min}$ of denaturation at $94^{\circ} \mathrm{C}\left(\mathrm{ramp}\right.$ rate $\left.4.4^{\circ} \mathrm{C} / \mathrm{s}\right)$, followed by 45 amplification cycles at $95^{\circ} \mathrm{C}$ for $10 \mathrm{~s}\left(4.4^{\circ} \mathrm{C} / \mathrm{s}\right), 60^{\circ} \mathrm{C}$ for $30 \mathrm{~s}\left(2.2^{\circ} \mathrm{C} / \mathrm{s}\right)$, and $72^{\circ} \mathrm{C}$ for $1 \mathrm{~s}\left(4.4^{\circ} \mathrm{C} / \mathrm{s}\right)$. The final step consisted of cooling the reaction mixture to $40^{\circ} \mathrm{C}\left(2.2^{\circ} \mathrm{C} / \mathrm{s}\right)$ with a $30 \mathrm{~s}$ hold. The fluorescent signal was measured on the FAM $(465-510 \mathrm{~nm})$ channel at the end of each step, at $72^{\circ} \mathrm{C}$.

The analytical sensitivity of the assay was determined as described previously [30] by testing triplicates of a 10-fold dilution series of HPV199 reference plasmid, ranging from $1 \times 10^{9}$ to $1 \times 10^{0} \mathrm{DNA}$ copies/reaction, and the detection limit was established to be at least ten viral copies. The standard curve of the assay was characterized by a high correlation coefficient $\left(\mathrm{R}^{2}=\right.$ 0.999), high amplification efficiency $(102.3 \%)$ and a dynamic range of eight orders of magnitude, enabling reliable discrimination of 10 to $10^{9} \mathrm{DNA}$ copies/reaction. For each sample panel tested, several negative RT-PCR controls consisting of water were included to check for possible amplicon carry-over contamination.

\section{Determination of the tissue tropism of HPV199}

To determine the tissue tropism of HPV199, a representative collection of various HPV-associated clinical specimens was tested with a HPV199 type-specific RT-PCR assay. In total, 916 samples obtained from the same number of immunocompetent individuals were tested: 76 tissue samples of histologically confirmed common warts, 108 samples of eyebrow hair follicles, 137 anal canal swabs obtained from individuals with clinically evident anal pathology, including anal warts, hemorrhoids and anal fissure, 184 nasopharyngeal swab samples and 411 cervical swabs obtained from women with normal cervical cytology. Total DNA was isolated from all samples using various, specimen-adjusted protocols, as described in detail previously [13, 31-33]. All clinical specimens were tested for human beta-globin to assess cell adequacy and the quality of the isolated DNA, and had to be positive to be included in the analysis. The presence of human beta-globin in cervical and anal swabs was determined by the internal control of the Linear Array HPV Genotyping Test (Linear Array; Roche Molecular Diagnostics, Pleasanton, CA), whereby an additional primer pair targeted $268 \mathrm{bp}$ of the human beta-globin gene. The presence of the human beta-globin in all other clinical specimens was determined using a slightly modified RT-PCR, targeting a 150 bp long fragment of the human beta-globin gene [34]. 


\section{Ethics Statement}

The present study was conducted in accordance with the Helsinki Declaration. All DNA samples tested for the presence of HPV199 DNA were obtained from our past or ongoing studies $[13,31,33,35]$ and none of the individuals were sampled solely for the objective of the present study. All studies from which samples for HPV199 testing were used have been approved by the National Medical Ethics Committee at the Slovenian Ministry of Health of the Republic of Slovenia (consent numbers as follows: 131/06/07, 45/04/07, 83/11/09, 109/08/12 and 63/10/ 13). All involved patients and individuals provided standardized written informed consents allowing testing for a broad range of HPVs for research purposes. In order to keep patient identities confidential, all samples were coded and only clinicians were able to link an individual's identity with the assigned study number and HPV199 results. The Institutional Review Board of the Institute of Microbiology and Immunology, Faculty of Medicine, University of Ljubljana, specifically approved the protocol of the present study and the use of stored DNA samples for the HPV199 testing.

\section{Results and Discussion}

HPV199 was originally found in a nasopharyngeal swab sample obtained from a 25 year-old immunocompetent male with a clinical suspicion of Bordetella pertussis infection. A partial 331 bp L1 sequence (Acc. no HG515499), suggesting the presence of a putative novel GammaPV type, was initially obtained with FAP6085F/FAP64 primers [15, 16], using a previously published PCR protocol [17]. Nasopharyngeal swab sample additionally tested positive for HPV5, HPV8 and HPV12 with primer sets targeting a broad spectrum of HPV types belonging to the Alpha-PV, Beta-PV and Gamma-PV genera [15-17]. The complete viral genome was amplified using HPV199 type-specific inverse long range PCR and cloned into a plasmid vector, and sequenced using a primer walking strategy. In order to determine possible artifacts in the cloned viral genome, the corresponding amplicon used for HPV199 cloning was additionally completely sequenced. Namely, it has been shown that alterations during the cloning process can occur, indicating the possibility that the cloned sequence is not an authentic copy of the genomic sequence [36, 37]. Since 100\% identity in the cloned HPV199 DNA sequence and viral genomic HPV199 DNA was determined by both methods, the generation of a cloning artifact due to the cloning process is highly unlikely [36-38]. In May 2014, the reference clone and corresponding nucleotide sequence were submitted to the Human Papillomavirus Reference Center at the Karolinska Institute in Sweden, where its nucleotide sequence was reconfirmed and assigned the official number HPV199 in July 2014 (http://www.hpvcenter.se/html/ refclones.html). The complete genome sequence was deposited in GenBank under accession number KJ913662.

In order to confirm uniqueness of the HPV199 we determined a complete nucleotide sequence of one additional HPV199 DNA isolate. The additional HPV199 complete viral genome was amplified using HPV199 type-specific inverse long range PCR and sequenced using primer walking strategy from anal canal swab sample obtained from 39-year old immunocompetent male. The complete viral genome of the second HPV199 DNA isolate exhibits 99.4\% nucleotide identity with the original HPV199 isolate, suggesting new variant of the novel HPV199. The complete genome sequence of this isolate was deposited in GenBank under accession number KT372348.

As shown in Fig 1, the complete genome of HPV199 is 7,184 bp in length with a GC content of $36.5 \%$. It demonstrates the typical genomic organization of Gamma-PVs, with the seven classical major open reading frames (ORFs), encoding five early (E1, E2, E4, E6 and E7) and 


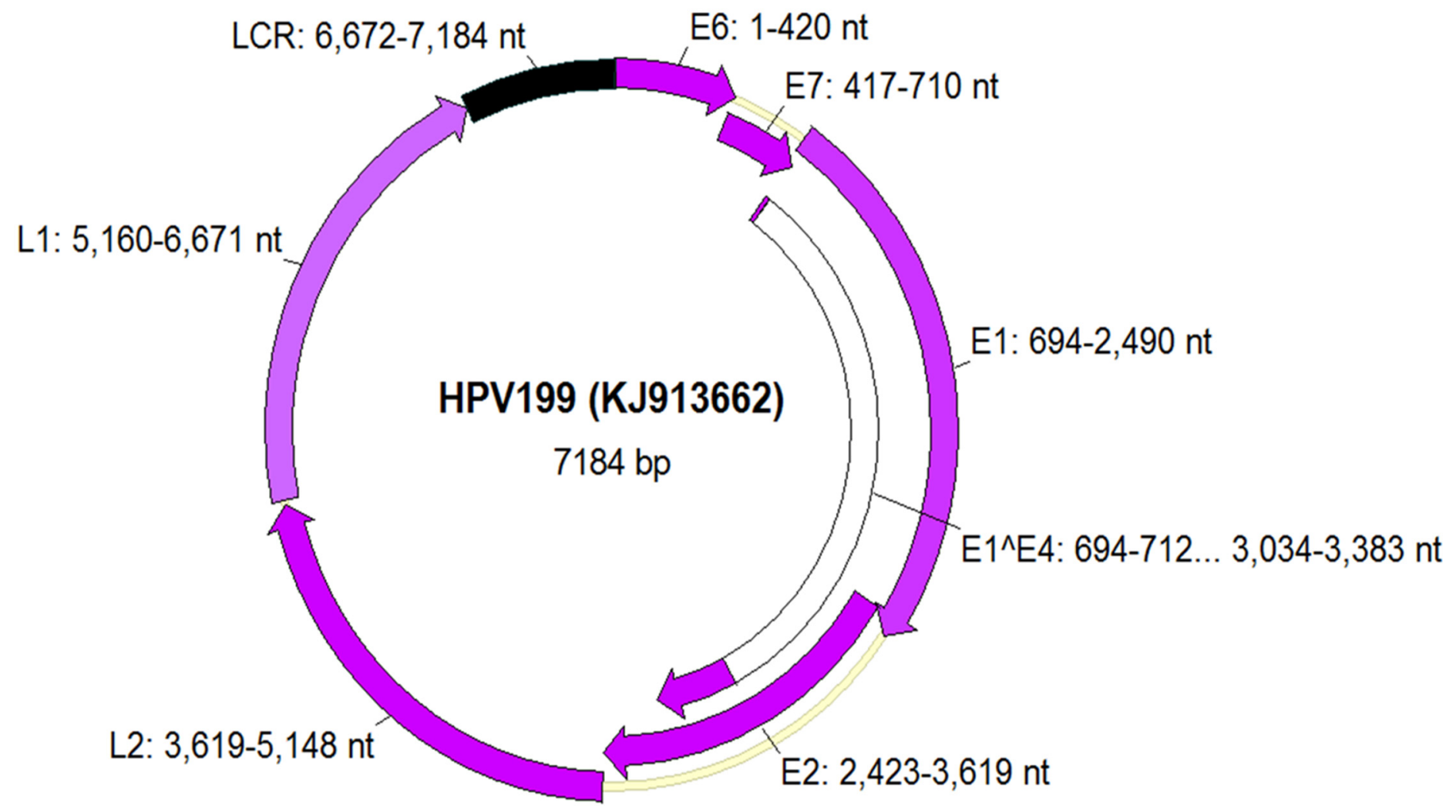

Fig 1. Genomic organization of HPV199 showing genomic positions of viral genes E6, E7, E1, E2, E4, L1, L2 and the non-coding long control region (LCR) located between L1 and E6.

doi:10.1371/journal.pone.0138628.g001

two late proteins (L1 and L2). As expected for Gamma-PVs, none of the other small ORFs identified in the genome of HPV199 showed significant similarity to known E5 proteins [39].

The first position of the nucleotide sequence of the whole genome of HPV199 corresponds to the first ATG of the putative E6 protein, which contains two conserved zinc-finger domains $\left(\mathrm{CxxC}(\mathrm{x})_{29} \mathrm{CxxC}\right)$ that are separated by 36 amino acids. The putative $\mathrm{E} 7$ protein that is located downstream of E6 ORF contains one zinc-finger domain (Fig 2). As additionally shown in Fig 2, these particular domains were also identified in E6 and E7 proteins of all other members of the species Gamma-12. Zinc-finger domains seem to be essential structures in the formation of multimerized protein complexes [40-42]. As with several other HPV types, HPV199 E7 protein contains no binding domain $(\mathrm{LxCxE})$ for the conserved retinoblastoma tumor suppressor protein ( $\mathrm{pRB}$ ). This conserved motif is necessary and sufficient for the association between E7 and pRB [43], although it has been shown that some types lacking the LxCxE motif cluster together in a phylogenetic tree, suggesting the motif was lost in the ancestral HPV of the cluster [44]. On the other hand, our analysis showed that four members of the same species (HPV132, HPV148, HPV165 and CG2) contain a pRB binding motif in the N-terminal part of the E7 protein (Fig 2). These results are consistent with results published earlier for HPV132 and HPV148 [45].

One conserved ATP-binding site of the ATP-dependent DNA helicase with a consensus sequence (GXXXXGK(T/S) was identified at aa 426-433 in the largest protein of HPV199 E1, with 598 amino acids [46]. This particular domain was additionally identified in E1 proteins of all other members of the species Gamma-12 (S1 Fig). When expressed, E1 accumulates 

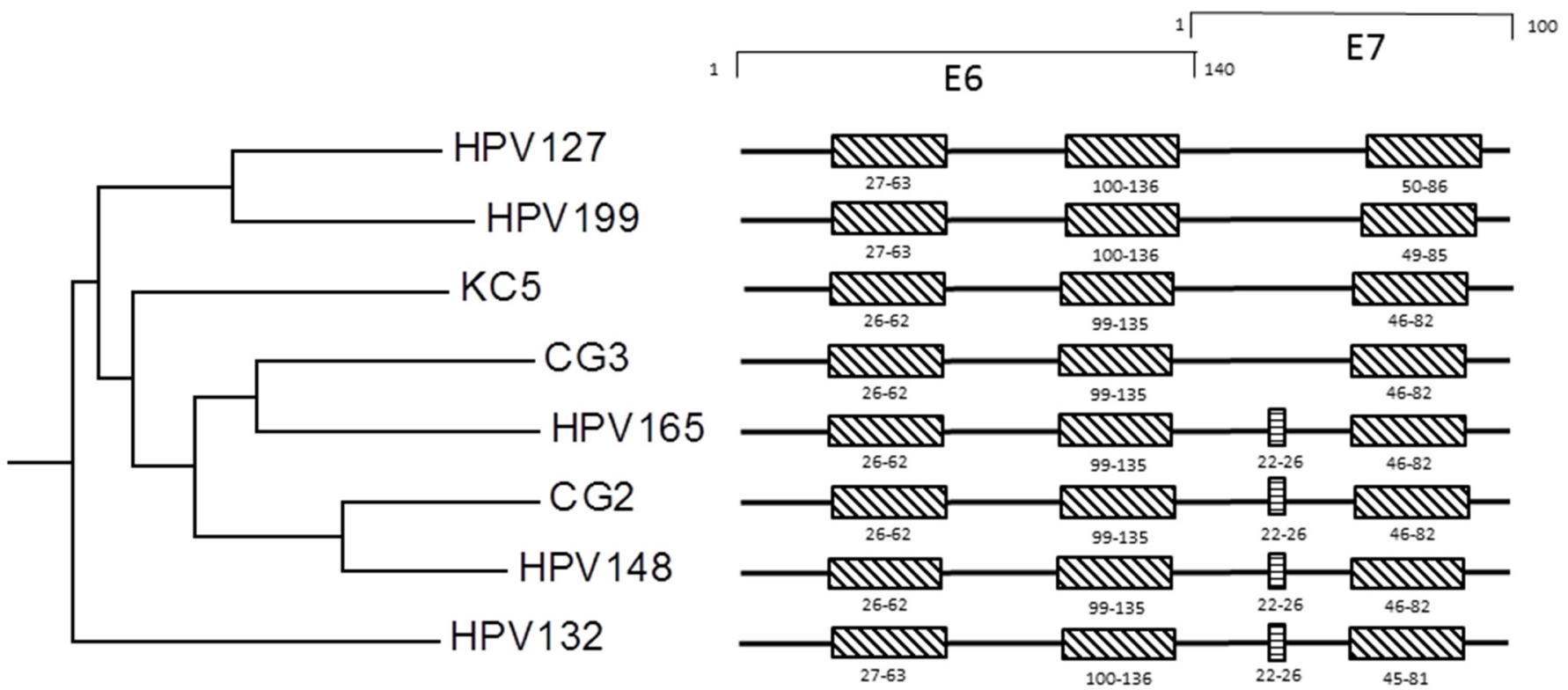

Fig 2. Localization of zinc-finger domains and binding domain for conserved retinoblastoma tumor suppressor protein (pRb) in E6 and E7 ORF of HPV199 and phylogenetically related HPV types. The E6 and E7 ORFs of all HPV types belonging to Gamma-PV species 12 contain two and one zincfinger domains (CxxC(x)29CxxC), respectively. The pRB binding motif (LxCxE) was identified only in the E7 ORF of HPV165, CG2, HPV148 and HPV132. Locations of zinc-finger domains are marked as boxes with oblique lines and locations of $p R b$ binding domains as boxes with horizontal lines.

doi:10.1371/journal.pone.0138628.g002

primarily in the nucleus of transfected cells. Nevertheless, studies on HPV11, HPV31 and BPV1 E1 have demonstrated that, although this protein is predominantly found in the nucleus at the steady-state, it can shuttle between the nucleus and cytoplasm via distinct nuclear localization and export signals [47].

Consequently, a typical bipartite nuclear localization signal (NLS), composed of two clusters of aa (KRK and KRRL) separated by 27 aa, was identified at the HPV199 E1 n-terminal part at codons 80-113 (nt, 928-1029) (S2 Fig). Similarly, a leucine-rich nuclear export signal (NES) $\left(\mathrm{L}-\mathrm{X}_{(2-3)}-\mathrm{L}-\mathrm{X}_{2}-(\mathrm{L} / \mathrm{I} / \mathrm{V})-\mathrm{X}-(\mathrm{L} / \mathrm{I})\right)$, typically recognized by the cell Crm1 exportin [47], was identified within the NLS region of the HPV199 E1 protein at codons 94-103 (nt, 970-999) (S2 Fig). As shown in S2 Fig, both NLSs and NESs were also successfully identified in all relatives of HPV199, with some minor differences in the aa composition.

No conserved leucine zipper domain $\left(\mathrm{L}-\mathrm{X}_{6}-\mathrm{L}-\mathrm{X}_{6}-\mathrm{L}-\mathrm{X}_{6}-\mathrm{L}\right)$ is present in the carboxyterminal part of the putative E2 protein of HPV199. A highly conserved NLS motif (RKRXR), which is present in many of the Alpha-HPVs and which in HPV6 and HPV11 promotes nuclear localization and association of the E2 proteins with the nuclear matrix [48], was identified at codons 231-235 (S3 Fig). As additionally shown in S3 Fig, the same conserved or slightly modified NLS motif was identified in E2 proteins of all other members of the species Gamma-12.

The HPV199 E4 ORF is typically positioned within the E2 ORF (nt, 2,997-3,383) and has its own start codon. However, due to the presence of the characteristic donor (AAG/GUASNR) and acceptor (GUYACYAG/YU) RNA splicing sites in E1 and E2 ORFs, respectively, [5, 49] it is most likely that the $\mathrm{E} 4$ protein is translated from a spliced mRNA, containing the first few codons of the E1 ORF joined to the E4 sequence (Fig 1, E1^E4, coding sequence consisting of genomic positions nt $694 \ldots 712$ and 3,034 . .3,383), as described previously for other HPV types $[22,50]$. We observed a typically high proline content in the E1^${ }^{\wedge} 4$ protein, with 20 proline residues out of 128 amino acids (15.6\%). 
The HPV199 late genomic region encodes two structural proteins: the major (L1) and minor (L2) capsid proteins. Assembly of PV virions occurs in the cell nucleus and a highly conserved polybasic patch at (or very near) the C-terminus of L1 and L2 has been identified as directing their import into the nucleus [51-54]. Consequently, NLS-like signals were identified at the C-terminal part of L1 and L2 proteins of HPV199 and all other members of the species Gamma-12 (S4 and S5 Figs) [51, 52]. In addition, the N-terminal part of the L2 protein of all Gamma-12 HPVs (except HPV165) contains a highly conserved furin cleavage motif (R-X-K/ R-R) (S6 Fig). The cleavage of the HPV L2 protein by cellular furin protease results in viral capsid conformational changes, leading to internalization of the virions into the basal cells to deliver the viral genome to their nucleus [53]. HPV165 probably has a differently shaped capsid, which does not require a second conformational change and therefore lacks the furin cleavage motif. Following viral dissociation, the viral DNA must escape the late endosome to be able to travel to the nucleus [53]. It has recently been suggested that a transmembrane domain located near the $\mathrm{N}$-terminus of the $\mathrm{L} 2$ protein probably aids vesicular compartment escape of the L2/HPV-DNA complex $[53,55]$. In line with this observation, the L2 transmembrane domain-like aa sequence (IVYFGGLGIGSGKGSG) was identified in the HPV199 L2 protein at codons 49-67 (nt, 3,763-3,819) (S7 Fig). As shown in S7 Fig, this domain was further identified in L2 of all relatives of HPV199, with some minor differences in the aa composition. Lastly, at its 5' end, the HPV199 L2 ORF additionally contains a putative polyadenilation site (AATAAA; nt, 3,709-3,714) that is necessary for processing viral early gene transcripts. This particular genetic signature was further identified at the same 5 ' location within the L2 gene of all other Gamma-12 species members.

The HPV199 long control region (LCR) is positioned between L1 and E6 ORFs at nucleotide positions 6,672 to 7,184 (513 bp) and contains four consensus palindromic E2-binding sites (ACC-N - $-7^{-G G T}$; nt 6,916-6,927, nt 6,957-6,969, nt 7,064-7,075, nt 7,133-7,144), a putative polyadenilation site (AATAAA, nt 6,767-6,772) for L1/L2 gene transcripts, a putative E1 binding site (consensus sequence: CTCATAGTTGCCAACTATTAT, nt 7,093-7,113), probably representing the origin of replication (S8 Fig) [56], and binding sites for transcriptional regulatory factors such as AP-1, NF-1, Sp1, TFIID and C/EBP [56, 57]. A putative TATA box (TATAAA) of the E6 promoter was identified $31 \mathrm{nt}$ upstream of the first start codon of the E6 ORF [56, 57]. The same characteristics were examined in all other Gamma-12 species members and they exhibited similar genetic features of LCR as HPV199. The genetic characteristics of the LCR genomic region of all current and putative members of the species Gamma-12 are summarized in S2 Table.

Phylogenetic analysis revealed that HPV199 clusters into the Gamma-PV genus, species Gamma-12, which additionally contains HPV127, HV132, HPV148, HPV165, and three putative HPV types: KC5, CG2 and CG3 (Fig 3). An identical evolutionary relationship was observed in the phylogenetic tree based solely on the L1 nucleotide sequences. According to the topology of the constructed phylogenetic tree and PACS full-genome global alignment, HPV199 is most closely related to HPV127, with 77\% nucleotide identity in the entire L1 region and $73.21 \%$ in the entire genome. Further analysis additionally confirmed HPV127 as the closest relative of HPV199, with the highest similarities in all compared viral ORFs and proteins (Table 1). As shown in Table 1, pairwise comparison of the novel HPV199 with the remaining members of Gamma-12 identified nucleotide similarities of the L1 ORF, with values ranging from $67.8-69.5 \%$. The ratios of relatedness (L1:E1 ratio) were calculated within Gamma-12 members and are shown in Table 1. Values near 1.0 indicate that genomes within this species exhibit similar degrees of homology across different regions of their genomes [28, 29], thereby confirming their close phylogenetic relationship. 


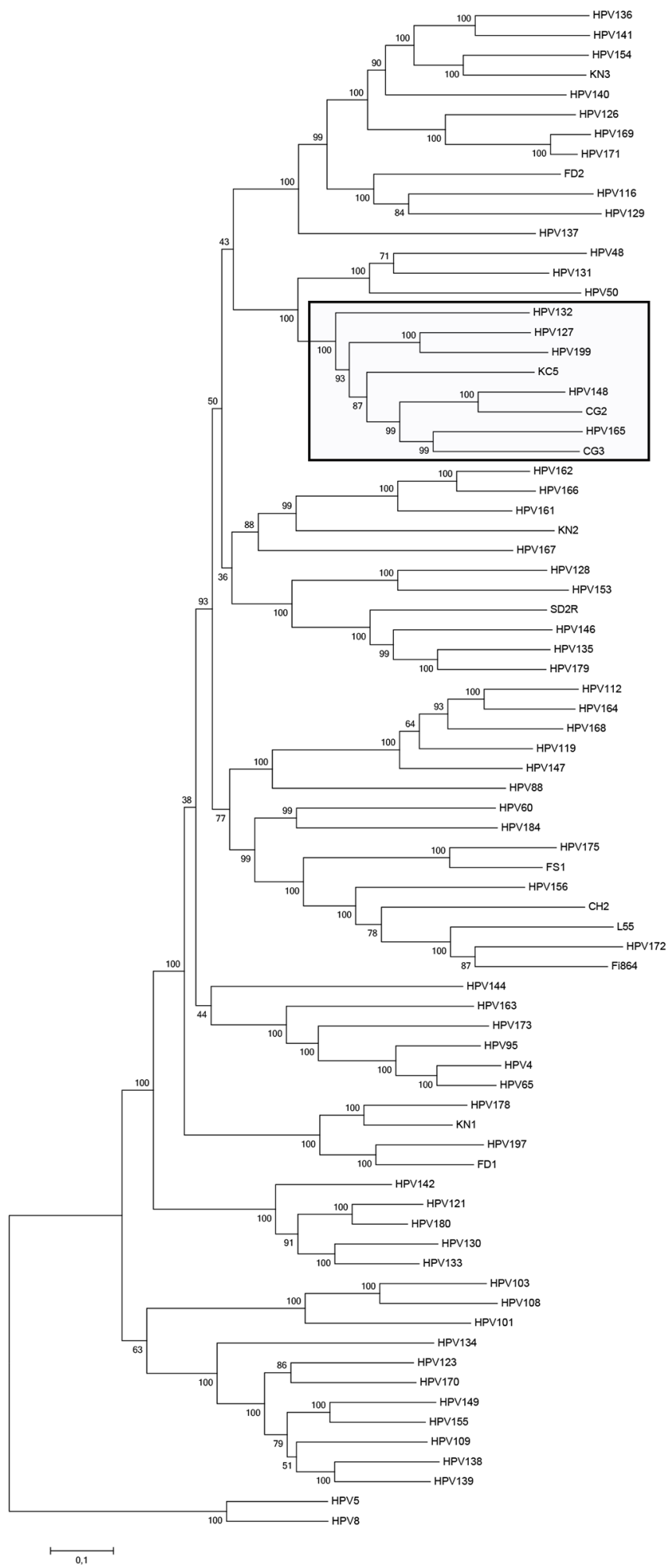

Fig 3. Phylogenetic analysis of HPV199. The complete HPV genome sequences of 75 officially recognized HPV types belonging to the genus Gamma-PV and all putative Gamma-PV types were obtained and aligned and a maximum likelihood phylogenetic tree was constructed. The nucleotide sequences of two Beta-PVs, HPV5 and HPV8, were used to root the tree. The numbers in each branch are bootstrap support values and are given as percentages. Species Gamma-12 is indicated by a black box.

doi:10.1371/journal.pone.0138628.g003 
Table 1. Nucleotide and amino acid sequence percentage similarities between individual genes (E6, E7, E1, E2, E4, L2 and L1) and the long control region (LCR) of HPV199 and closest HPV types.

\begin{tabular}{|c|c|c|c|c|c|c|c|c|c|c|}
\hline \multirow{2}{*}{\multicolumn{2}{|c|}{$\begin{array}{l}\text { HPV types } \\
\text { (Gamma-12) }\end{array}$}} & \multicolumn{8}{|c|}{ Pairwise similarity with HPV199 (\%) } & \multirow[t]{2}{*}{ L1:E1 ratio } \\
\hline & & E6 & E7 & E1 & E2 & E4 & L2 & L1 & LCR & \\
\hline \multirow[t]{2}{*}{ HPV127 } & $\mathrm{nt}$ & 83.2 & 74.6 & 78.6 & 76.5 & 76.7 & 64.6 & 77.0 & 50.0 & 0.980 \\
\hline & aa & 92.0 & 83.5 & 85.9 & 82.6 & 71.7 & 74.0 & 90.4 & - & 1.052 \\
\hline \multirow[t]{2}{*}{ HPV132 } & $\mathrm{nt}$ & 60.2 & 62.0 & 72.8 & 69.0 & 65.9 & 60.4 & 68.6 & 56.2 & 0.942 \\
\hline & aa & 72.1 & 64.6 & 82.1 & 75.0 & 66.7 & 69.6 & 79.4 & - & 0.967 \\
\hline \multirow[t]{2}{*}{ HPV148 } & $\mathrm{nt}$ & 65.0 & 61.7 & 71.9 & 66.7 & 64.6 & 61.8 & 68.1 & 53.2 & 0.947 \\
\hline & aa & 70.5 & 76.0 & 79.4 & 72.1 & 58.3 & 70.4 & 81.6 & - & 1.028 \\
\hline \multirow[t]{2}{*}{ HPV165 } & $\mathrm{nt}$ & 72.0 & 65.6 & 72.0 & 65.9 & 67.5 & 59.2 & 68.2 & 49.8 & 0.947 \\
\hline & aa & 83.0 & 76.8 & 79.0 & 70.7 & 60.8 & 68.0 & 78.3 & - & 0.991 \\
\hline \multirow[t]{2}{*}{ CG2 } & $\mathrm{nt}$ & 66.8 & 58.5 & 70.8 & 66.0 & 66.7 & 60.2 & 67.9 & 53.5 & 0.959 \\
\hline & aa & 70.9 & 75.0 & 79.8 & 70.4 & 58.3 & 72.1 & 81.1 & - & 1.016 \\
\hline \multirow[t]{2}{*}{ CG3 } & nt & 69.0 & 63.2 & 73.2 & 64.6 & 64.4 & 60.0 & 67.8 & 52.0 & 0.926 \\
\hline & aa & 79.3 & 78.1 & 82.1 & 72.3 & 59.8 & 68.9 & 79.9 & - & 0.973 \\
\hline \multirow[t]{2}{*}{ KC5 } & $\mathrm{nt}$ & 68.5 & 68.2 & 74.6 & 68.0 & 67.4 & 61.2 & 69.5 & 53.9 & 0.932 \\
\hline & aа & 78.5 & 74.5 & 84.1 & 72.6 & 63.4 & 71.7 & 82.8 & - & 0.985 \\
\hline
\end{tabular}

doi:10.1371/journal.pone.0138628.t001

Recent studies have detected Gamma-PVs at various sites, including healthy skin and various cutaneous lesions [6-9] and genital [10], anal [12], oral [4] and nasal [11] mucosa, suggesting that the original perception of Gamma-PVs as cutaneous HPV types was too narrow [5]. In contrast, members of Gamma-12 have so far been found predominantly in healthy skin samples and skin warts [5]. Specifically, HPV127, HPV132 and HPV165 were originally detected in skin swab samples obtained from healthy individuals, indicating that these viruses are probably skin commensals $[9,58,59]$. Additionally, recent metagenomic analysis using shotgun sequencing approach identified three Gamma-12 members HPV127, HPV132 and HPV148 in three, four and seven skin samples, obtained from healthy individuals, respectively [8]. In the same study HPV148 was also found in a single oral mucosa sample obtained from a healthy individual [8]. In recently published study, HPV132 and HPV148 were occasionally detected in anal samples obtained from HIV-positive and HIV-negative men who have sex with men [60]. On the other hand, the persistence of HPV132 and HPV148 in common skin warts and histologically confirmed actinic keratotic lesions, respectively, suggests that some members of Gamma-12 may be implicated in the development of benign and precancerous lesions in immunosuppressed individuals [45]. Three putative HPV types (CG2, CG3, KC5) that were preliminary classified into Gamma-12 [5], have been found in normal skin, while CG2 was additionally found in a skin sample of a female renal transplant recipient $[7,59,61]$.

In order to assess HPV199 tissue tropism, various clinical specimens representing known sites of HPV infection were tested using an HPV199 type-specific RT-PCR assay (Table 2). The sample collection included cutaneous samples, particularly eyebrow hair follicles, which are a known reservoir of Gamma-PVs [9,62] and common warts, which are cutaneous lesions with a productive HPV infection and which might be occasionally induced by Gamma-PVs [22]. Additionally, anal canal swabs, nasopharyngeal swabs and cervical swabs were tested to determine the prevalence of HPV199 in mucocutaneous and mucosal epithelia. As shown in Table 2, of 916 clinical samples tested, HPV199 DNA was detected in a total of 13 samples (1.4\%), indicating a low overall prevalence of HPV199 across different human epithelia, similar to other Gamma-PVs [5, 22, 63]. The presence of other HPV types which were previously determined with several in-house and commercial HPV broad-range PCR protocols in our 
Table 2. Detection of HPV199 in 916 clinical samples using an HPV199 type-specific RT-PCR assay.

\begin{tabular}{llccc}
\hline Tissue type & $\begin{array}{l}\text { Anatomical location (sample } \\
\text { type) }\end{array}$ & $\begin{array}{c}\text { Total number of samples } \\
\text { tested }\end{array}$ & $\begin{array}{c}\text { Number of HPV199 positive } \\
\text { samples }\end{array}$ & $\begin{array}{c}\text { Prevalence of } \\
\text { HPV199 }\end{array}$ \\
\hline Cutaneous & Common warts (tissue) & 76 & 2 & $2.6 \%$ \\
& Eyebrows (hair follicles) & 108 & 2 & $1.9 \%$ \\
Mucocutaneous & Anal canal (swabs) & 137 & 2 & $1.5 \%$ \\
Mucosal & Nasopharynx (swabs) & 184 & 4 & 3 \\
& Cervix (swabs) & 411 & 13 & $2.2 \%$ \\
Total & & 916 & $0.7 \%$ & $1.4 \%$ \\
\hline
\end{tabular}

doi:10.1371/journal.pone.0138628.t002

past or ongoing studies [13, 31, 33, 35] in all 916 clinical specimens used to determine HPV199 tissue tropism is summarized in $\mathrm{S} 3$ Table.

Although we detected HPV199 in two out of 76 (2.6\%) common warts tested, we were not able to establish an etiological link between the presence of HPV199 and the development of these two common warts, due to the detection of other established common wart-associated HPV types, particularly HPV2, in both warts. However, data from some studies have shown that Gamma-PVs can be etiologically linked with sporadic cases of common warts and actinic keratosis, especially in immunosuppressed patients [22, 45, 64-67], so further studies are needed to determine the potential clinical significance of HPV199. HPV199 was further detected in two out of 108 (1.9\%) samples of eyebrow hair follicles, confirming accumulating data about hair follicles as an important reservoir of various HPV types from different HPV genera [13, 22, 35, 68-70]. Additionally, JL and QD recently identified in a skin sample a partial L1 sequence 252 bp in length, with a 99\% nucleotide identity to HPV199 (Acc. No KC752084) [17] but all attempts to clone HPV199 from this sample failed.

Since HPV199 was originally detected in the nasopharynx, a total of 184 nasopharyngeal swabs were additionally tested and four (2.2\%) tested HPV199 positive. Our data are consistent with a recently published report indicating that Gamma-PVs exhibit tropism for nasal mucosa [11]. Although a relatively high prevalence of Beta-PV and Gamma-PV types was observed in this particular study, the potential clinical significance of Beta-PV and Gamma-PV types in the nasal region remains unknown [11].

HPV199 was also detected in two (1.5\%) out of 137 anal canal swabs tested. Similar results were reported in a recently published study in which the diversity of HPV in the male anal canal was investigated and a broad spectrum of HPV types belonging to the Alpha-PV, BetaPV and Gamma-PV genera was identified [12]. Although the prevalence and type-specific distributions of Gamma-PV (5.3\%) were significantly lower than in other HPV genera, this area requires further research.

To the best of our knowledge, the prevalence of Gamma-PVs in cervical samples obtained from women with normal cervical cytology had not been evaluated prior to this study. In the initial testing of 96 cervical swab samples obtained from women with normal cervical cytology, one sample tested positive for the presence of HPV199. Since none of the published reports have previously detected any member of Gamma-12 in the cervix, a larger number (total of 411 samples) of cervical samples were additionally tested, and two more samples tested HPV199 positive, producing a total HPV 199 prevalence of $0.7 \%$. So far, only a few HPV types belonging to Gamma-PV have been found in the cervix [5, 8, 29, 71]. Three related HPV types: HPV101, HPV103 and HPV108, all belonging to Gamma-6, were previously found in samples of highgrade cervical intraepithelial neoplasia, normal genital mucosa and low-grade cervical intraepithelial neoplasia, respectively $[29,71]$ but a possible etiological link between HPV infection and the development of a cervical intraepithelial lesion has been established only for HPV108 
[71]. A recent metagenomics analysis also revealed the presence of Gamma-PV in the anogenital region, more specifically vagina [8]. Thus, according to the results of our study and literature data, dual tropism of all Gamma-12 members is highly possible.

It has been shown previously that HPV types belonging to Gamma-12 can establish a persistent infection in immunosuppressed individuals [22, 45]. In our study, additional samples were available only for three HPV199 positive individuals but all three follow-up samples, obtained 35, 38 and 38 months after initial testing, respectively, tested HPV199 negative. Due to the low HPV199 initial prevalence and few available follow-up samples, we cannot draw a final conclusion about HPV199 persistence ability, so further studies on a larger number of samples are needed.

\section{Conclusions}

A novel HPV type, HPV199, was initially identified in a nasopharyngeal swab sample and successfully characterized. The complete genome of HPV199 has a length of 7,184 bp and contains five early (E1, E2, E4, E6 and E7) and two late (L1 and L2) ORFs, but no E5 ORF, a genomic organization typical of other Gamma-PVs. Comparative characterization of HPV199 and its closest relatives and phylogenetic analysis confirmed that HPV-199 clusters into the GammaPV genus, species Gamma-12, additionally containing HPV127, HV132, HPV148, HPV165, and three putative HPV types: KC5, CG2 and CG3. The complete viral genome of additional HPV199 isolate was sequenced from anal canal swab sample. Two independent HPV199 complete viral sequences exhibit $99.4 \%$ nucleotide identity. To the best of our knowledge, this is the first member of Gamma-PV with complete nucleotide sequences determined from two independent clinical samples. Overall, HPV199 was found in 1.4\% of cutaneous and mucosal samples tested only, but it exhibits dual tissue tropism. According to the results of our study and literature data, dual tropism of all Gamma-12 members is highly possible.

\section{Supporting Information}

S1 Fig. Conserved ATP-binding site of the ATP-dependent DNA helicase with consensus sequence (GXXXXGK(T/S) identified in E1 proteins of all Gamma-12 members. Consensus sequences are indicated by a red box.

S2 Fig. Typical bipartite nuclear localization signal (NLS) and nuclear export signal (NES) identified in E1 proteins of all Gamma-12 members. NLS, composed of two clusters of aa (KRK and KRRL), is indicated with black boxes and NES with a red box.

S3 Fig. Highly conserved nuclear localization signal (NLS) motif (RKRXR) in E2 proteins of all Gamma-12 members. A conserved or slightly modified NLS motif is indicated with red box.

S4 Fig. Nuclear localization-like signals (NLS-like) at C-terminal part of L1 proteins of all Gamma-12 members. NLS-like signals are indicated with a red box. (TIF)

S5 Fig. Nuclear localization-like signals (NLS-like) at the C-terminal part of L2 proteins of all Gamma-12 members. NLS-like signals are indicated with a red box.

(TIF) 
S6 Fig. Highly conserved furin cleavage motif $(\mathrm{R}-\mathrm{X}-\mathrm{K} / \mathrm{R}-\mathrm{R})$ at the $\mathrm{N}$-terminal part of $\mathrm{L} 2$ proteins of all Gamma-12 members. Furin cleavage motifs are indicated with a red box. (TIF)

S7 Fig. L2 transmembrane domain-like aa sequence in L2 proteins of all Gamma-12 members. $\mathrm{L} 2$ transmembrane domain-like sequences with some minor differences in aa composition are indicated with a red box.

S8 Fig. Putative E1-binding sites identified in the long control region (LCR) of all Gamma12 members. A total of $144 \mathrm{HPV}$ reference sequences was obtained from the Papillomavirus Episteme database (PaVE) and aligned with the Pro-Coffee algorithm of the T-coffee multiple sequence alignment package (http://tcoffee.crg.cat/apps/tcoffee/do:procoffee). The obtained alignment was visualized with the JalView 2.8.1 software package and colored based on the percentage identify option. The E1-binding site sequence logo was derived from the alignment and generated with WebLogo 2.8.2 using the web server at http://weblogo.berkeley.edu/logo. cgi.

S1 Table. Primers used for HPV-199 whole genome sequencing using a primer walking strategy.

(DOCX)

S2 Table. Summary of genetic features of the LCR genomic region of current and putative members of species Gamma-12.

(DOCX)

S3 Table. HPV types determined in 916 various clinical specimens using an HPV199 typespecific real-time PCR assay and different primer sets targeting a broad spectrum of HPV types belonging to Alpha-PV, Beta-PV and Gamma-PV. (DOCX)

\section{Acknowledgments}

We are grateful to Professor Katja Seme, $\mathrm{MD}, \mathrm{PhD}$ for helpful discussion and her invaluable support. We thank Jacques Archambault, $\mathrm{PhD}$ for his help with the determination of the putative E1 binding site.

\section{Author Contributions}

Conceived and designed the experiments: AO LH BJK MP. Performed the experiments: AO LH AŠ JL QD. Analyzed the data: AO LH AŠ BJK. Contributed reagents/materials/analysis tools: MP JL QD. Wrote the paper: AO AŠ BJK MP.

\section{References}

1. Bernard HU, Burk RD, Chen Z, van Doorslaer K, Zur Hausen H, de Villiers EM. Classification of papillomaviruses (PVs) based on 189 PV types and proposal of taxonomic amendments. Virology. 2010; 401: 70-79. doi: 10.1016/j.virol.2010.02.002 PMID: 20206957

2. Cubie HA. Diseases associated with human papillomavirus infection. Virology. 2013; 445: 21-34. doi: 10.1016/j.virol.2013.06.007 PMID: 23932731

3. Bzhalava D, Eklund C, Dillner J. International standardization and classification of human papillomavirus types. Virology. 2015; 476: 341-344. doi: 10.1016/j.virol.2014.12.028 PMID: 25577151 
4. Bottalico D, Chen Z, Dunne A, Ostoloza J, McKinney S, Sun C, et al. The oral cavity contains abundant known and novel human papillomaviruses from the Betapapillomavirus and Gammapapillomavirus genera. J Infect Dis. 2011; 204: 787-792. doi: 10.1093/infdis/jir383 PMID: 21844305

5. Ure $\mathrm{AE}$, Forslund $\mathrm{O}$. Characterization of human papillomavirus type 154 and tissue tropism of gammapapillomaviruses. PLoS One. 2014; 9: e89342. doi: 10.1371/journal.pone.0089342 PMID: 24551244

6. Ekström J, Bzhalava D, Svenback D, Forslund O, Dillner J. High throughput sequencing reveals diversity of Human Papillomaviruses in cutaneous lesions. Int J Cancer. 2011; 129: 2643-2650. doi: 10. 1002/ijc.26204 PMID: 21630257

7. Foulongne V, Sauvage V, Hebert C, Dereure O, Cheval J, Gouilh MA, et al. Human skin microbiota: high diversity of DNA viruses identified on the human skin by high throughput sequencing. PLoS One. 2012; 7: e38499. doi: 10.1371/journal.pone.0038499 PMID: 22723863

8. Ma Y, Madupu R, Karaoz U, Nossa CW, Yang L, Yooseph S, et al. Human papillomavirus community in healthy persons, defined by metagenomics analysis of human microbiome project shotgun sequencing data sets. J Virol. 2014; 88: 4786-4797. doi: 10.1128/JVI.00093-14 PMID: 24522917

9. Antonsson A, Erfurt C, Hazard K, Holmgren V, Simon M, Kataoka A, et al. Prevalence and type spectrum of human papillomaviruses in healthy skin samples collected in three continents. J Gen Virol. 2003; 84: 1881-1886. PMID: 12810883

10. Sichero L, Pierce Campbell CM, Ferreira S, Sobrinho JS, Luiza Baggio M, Galan L, et al; for the HIM Study Group. Broad HPV distribution in the genital region of men from the HPV infection in men (HIM) study. Virology. 2013; 443: 214-217. doi: 10.1016/j.virol.2013.04.024 PMID: 23722104

11. Forslund $\mathrm{O}$, Johansson $\mathrm{H}$, Madsen $\mathrm{KG}$, Kofoed $\mathrm{K}$. The nasal mucosa contains a large spectrum of human papillomavirus types from the betapapillomavirus and gammapapillomavirus genera. $\mathrm{J}$ Infect Dis. 2013; 208: 1335-1341. doi: 10.1093/infdis/jit326 PMID: 23878325

12. Sichero L, Nyitray AG, Nunes EM, Nepal B, Ferreira S, Sobrinho JS, et al. Diversity of human papillomavirus in the anal canal of men: the HIM study. Clin Microbiol Infect. 2015; 21: 502-509. doi: 10.1016/ j.cmi.2014.12.023 PMID: 25698660

13. Kocjan BJ, Poljak M, Seme K, Potočnik M, Fujs K, Babic DZ. Distribution of human papillomavirus genotypes in plucked eyebrow hairs from Slovenian males with genital warts. Infect Genet Evol. 2005; 5: 255-259. PMID: 15737917

14. Odar K, Kocjan BJ, Hošnjak L, Gale N, Poljak M, Zidar N. Verrucous carcinoma of the head and necknot a human papillomavirus-related tumour? J Cell Mol Med. 2014; 18: 635-645. doi: 10.1111/jcmm. 12211 PMID: 24350715

15. Forslund $\mathrm{O}$, Antonsson $A$, Nordin $P$, Stenquist $B$, Hansson $B G$. A broad range of human papillomavirus types detected with a general PCR method suitable for analysis of cutaneous tumours and normal skin. J Gen Virol. 1999; 80: 2437-2443. PMID: 10501499

16. Forslund $\mathrm{O}$, Ly H, Higgins $\mathrm{G}$. Improved detection of cutaneous human papillomavirus DNA by single tube nested 'hanging droplet' PCR. J Virol Methods. 2003; 110: 129-136. PMID: 12798239

17. Li J, Pan $Y, X u Z$, Wang Q, Hang D, Shen N, et al. Improved detection of human papillomavirus harbored in healthy skin with FAP6085/64 primers. J Virol Methods. 2013; 193: 633-638. doi: 10.1016/j. jviromet.2013.06.026 PMID: 23871757

18. Hall TA. BioEdit: a user-friendly biological sequence alignment editor and analysis program for Windows 95/98/NT. Nucleic Acids Symposium Series. 1999; 41: 95-98.

19. Rector A, Tachezy R, Van Ranst M. A sequence-independent strategy for detection and cloning of circular DNA virus genomes by using multiply primed rolling-circle amplification. J VIrol. 2004; 78: 49934998. PMID: 15113879

20. Platt AR, Woodhall RW, George AL Jr. Improved DNA sequencing quality and efficiency using an optimized fast cycle sequencing protocol. Biotechniques. 2007; 43: 58-62. PMID: 17695253

21. Tamura K, Stecher G, Peterson D, Filipski A, Kumar S. MEGA6: Molecular evolutionary genetics analysis version 6.0. Mol Biol Evol. 2013; 30: 2725-9. doi: 10.1093/molbev/mst197 PMID: 24132122

22. Hošnjak L, Kocjan BJ, Pirš B, Seme K, Poljak M. Characterization of Two Novel Gammapapillomaviruses, HPV179 and HPV184, Isolated from Common Warts of a Renal-Transplant Recipient. PLoS One. 2015; 10: e0119154. doi: 10.1371/journal.pone.0119154 PMID: 25748516

23. Van Doorslaer K, Tan Q, Xirasagar S, Bandaru S, Gopalan V, Mohamoud Y, et al. The Papillomavirus Episteme: a central resource for papillomavirus sequence data and analysis. Nucleic Acids Research 2013; 41: D571-578. Available: pave.niaid.nih.gov. Accessed 30 May 2015. doi: 10.1093/nar/gks984 PMID: 23093593

24. Edgar RC. MUSCLE: a multiple sequence alignment method with reduced time and space complexity. BMC Bioinformatics. 2004a; 5: 113. 
25. Edgar RC. MUSCLE: multiple sequence alignment with high accuracy and high throughput. Nucleic Acids Res. 2004b; 32: 1792-1797.

26. Chen Z, Schiffman M, Herrero R, Desalle R, Burk RD. Human papillomavirus (HPV) types 101 and 103 isolated from cervicovaginal cells lack an E6 open reading frame (ORF) and are related to gamma-papillomaviruses. Virology. 2007; 360: 447-453. PMID: 17125811

27. Bao Y, Chetvernin V, Tatusova T. Improvements to pairwise sequence comparison (PASC): a genome-based web tool for virus classification. Arch Virol. 2014; 159: 3293-3304. doi: 10.1007/ s00705-014-2197-x PMID: 25119676

28. Terai M, Burk RD. Identification and characterization of 3 novel genital human papillomaviruses by overlapping polymerase chain reaction: candHPV89, candHPV90, and candHPV91. J Infect Dis. 2002; 185: 1794-1797. PMID: 12085327

29. Chen Z, Schiffman M, Herrero R, Burk RD. Identification and characterization of two novel human papillomaviruses (HPVs) by overlapping PCR: HPV102 and HPV106. J Gen Virol. 2007; 88: 2952-2955. PMID: 17947516

30. Kocjan BJ, Poljak M, Seme K. Universal ProbeLibrary based real-time PCR assay for detection and confirmation of human papillomavirus genotype 52 infections. J Virol Methods. 2010; 163: 492-494 doi: 10.1016/j.jviromet.2009.10.024 PMID: 19883693

31. Milošević M, Poljak M, Mlakar B. Anal HPV infection in Slovenian men who have sex with men. Cent Eur J Med. 2010; 5: 698-703.

32. Fujs Komloš K, Košorok P, Kocjan BJ, Poljak M. Genetic diversity of HPV-6 in concurrent multiple anogenital warts. Acta Dermatovenerol Alp Pannonica Adriat. 2013; 22: 31-33. PMID: 23674183

33. Poljak M, Ostrbenk A, Seme K, Ucakar V, Hillemanns P, Bokal EV, et al. Comparison of clinical and analytical performance of the Abbott Realtime High Risk HPV test to the performance of hybrid capture 2 in population-based cervical cancer screening. J Clin Microbiol. 2011; 49: 1721-1729. doi: 10.1128/ JCM.00012-11 PMID: 21430098

34. van Duin M, Snijders PJ, Schrijnemakers HF, Voorhorst FJ, Rozendaal L, Nobbenhuis MA. Human papillomavirus 16 load in normal and abnormal cervical scrapes: an indicator of CIN II/III and viral clearance. Int J Cancer. 2002; 98: 590-595. PMID: 11920620

35. Potočnik M, Kocjan BJ, Seme K, Luzar B, Babič DZ, Poljak M. Beta-papillomaviruses in anogenital hairs plucked from healthy individuals. J Med Virol. 2006; 78: 1673-1678. PMID: 17063512

36. Kasher MS, Roman A. Alterations in the regulatory region of the human papillomavirus type 6 genome are generated during propagation in Escherichia coli. J Virol. 1988; 62: 3295-3300. PMID: 2841482

37. Heinzel PA, Chan SY, Ho L, O'Connor M, Balaram P, Campo MS, et al. Variation of human papillomavirus type 6 (HPV-6) and HPV-11 genomes sampled throughout the world. J Clin Microbiol. 1995; 33: 1746-1754. PMID: 7665641

38. Kocjan BJ, Poljak M, Cimerman M, Gale N, Potocnik M, Bogovac Z, et al. Prevaccination genomic diversity of human papillomavirus genotype 6 (HPV 6). Virology. 2009; 391: 274-283. doi: 10.1016/j. virol.2009.06.030 PMID: 19596128

39. Venuti A, Paolini F, Nasir L, Corteggio A, Roperto S, Campo MS, et al. Papillomavirus E5: the smallest oncoprotein with many functions. Mol Cancer. 2011; 10: 140. doi: 10.1186/1476-4598-10-140 PMID: 22078316

40. Narechania A, Terai M, Chen Z, DeSalle R, Burk RD. Lack of the canonical pRB-binding domain in the E7 ORF of artiodactyl papillomaviruses is associated with the development of fibropapillomas. J Gen Virol. 2004; 85: 1243-1250. PMID: 15105541

41. Ogawa $T$, Tomita $Y$, Okada M, Shirasawa H. Complete genome and phylogenetic position of bovine papillomavirus type 7. J Gen Virol. 2007; 88: 1934-1938. PMID: 17554025

42. Roman A, Munger K. The papillomavirus E7 proteins. Virology. 2013; 445: 138-168. doi: 10.1016/j. virol.2013.04.013 PMID: 23731972

43. McLaughlin-Drubin ME, Münger K. The human papillomavirus E7 oncoprotein. Virology. 2009; 384: 335-344. doi: 10.1016/j.virol.2008.10.006 PMID: 19007963

44. Mitsuishi T, Ohsawa I, Kato T, Egawa N, Kiyono T. Molecular cloning and characterisation of a novel type of human papillomavirus 160 isolated from a flat wart of an immunocompetent patient. PLoS One. 2013; 8: E79592. doi: 10.1371/journal.pone.0079592 PMID: 24282509

45. Köhler A, Gottschling M, Manning K, Lehmann M, Schulz E, Krüger-Corcoran D, et al. Genomic characterization of ten novel cutaneous human papillomaviruses from keratotic lesions of immunosuppressed patients. J Gen Virol. 2011; 92: 1585-1594. doi: 10.1099/vir.0.030593-0 PMID: 21471318

46. Titolo S, Pelletier A, Sauvé F, Brault K, Wardrop E, White PW, et al. Role of the ATP-binding domain of the human papillomavirus type $11 \mathrm{E} 1$ helicase in E2-dependent binding to the origin. J Virol. 1999; 73 : 5282-5293. PMID: 10364274 
47. Bergvall M, Melendy T, Archambault J. The E1 proteins. Virology. 2013; 445: 35-56 doi: 10.1016/j.virol. 2013.07.020 PMID: 24029589

48. Zou N, Lin BY, Duan F, Lee KY, Jin G, Guan R, et al. The hinge of the human papillomavirus type 11 E2 protein contains major determinants for nuclear localization and nuclear matrix association. J Virol. 2000; 74: 3761-3770. PMID: 10729151

49. Doorbar J, Myers G. The E4 Protein. In: Myers G, Halpern A, Baker C, McBride A, Wheeler C, Doorbar J, editors. H96 Compendium, vol. III. Los Alamos: Los Alamos National Laboratory; 1996. pp. 58-80.

50. Kovanda A, Kocjan BJ, Potočnik M, Poljak M. Characterization of a novel cutaneous human papillomavirus genotype HPV-125. PLoS One. 2011; 6: e22414. doi: 10.1371/journal.pone.0022414 PMID: 21811601

51. Zhou J, Doorbar J, Sun XY, Crawford LV, McLean CS, Frazer IH. Identification of the nuclear localization signal of human papillomavirus type 16 L1 protein. Virology. 1991; 185: 625-632. PMID: 1660197

52. Nelson LM, Rose RC, LeRoux L, Lane C, Bruya K, Moroianu J. Nuclear import and DNA binding of human papillomavirus type 45 L1 capsid protein. J Cell Biochem. 2000; 79: 225-238. PMID: 10967550

53. Wang JW, Roden RB. L2, the minor capsid protein of papillomavirus. Virology. 2013; 445: 175-186. doi: 10.1016/j.virol.2013.04.017 PMID: 23689062

54. Buck CB, Day PM, Trus BL. The papillomavirus major capsid protein L1. Virology. 2013; 445: 169-174. doi: 10.1016/j.virol.2013.05.038 PMID: 23800545

55. Bronnimann MP, Chapman JA, Park CK, Campos SK. A transmembrane domain and GxxxG motifs within L2 are essential for papillomavirus infection. J Virol. 2013; 87: 464-473. doi: 10.1128/JVI.0153912 PMID: 23097431

56. O'Connor M, Chan SY, Bernard HU. Transcription factor binding sites in the long control regions of genital HPVs. In: Myers G, Bernard HU, Delius H, Baker C, Icenogle J, Halpern A, Wheeler C, editors. Human Papillomaviruses 1995 Compendium. Los Alamos: Los Alamos National Laboratory; 1995. pp. 21-40.

57. Bernard HU. Regulatory elements in the viral genome. Virology. 2013; 445: 197-204. doi: 10.1016/j. virol.2013.04.035 PMID: 23725692

58. Schowalter RM, Pastrana DV, Pumphrey KA, Moyer AL, Buck CB. Merkel cell polyomavirus and two previously unknown polyomaviruses are chronically shed from human skin. Cell Host Microbe. 2010; 7: 509-515. doi: 10.1016/j.chom.2010.05.006 PMID: 20542254

59. Li J, Cai H, Xu Z, Wang Q, Hang D, Shen N, et al. Nine complete genome sequences of cutaneous human papillomavirus genotypes isolated from healthy skin of individuals living in rural He Nan province, China. J Virol. 2012; 86: 11936. doi: 10.1128/JVI.01988-12 PMID: 23043169

60. Torres M, Gheit T, McKay-Chopin S, Rodríguez C, Romero JD, Filotico R, et al. Prevalence of beta and gamma human papillomaviruses in the anal canal of men who have sex with men is influenced by HIV status. J Clin Virol. 2015; 67: 47-51. doi: 10.1016/j.jcv.2015.04.005 PMID: 25959158

61. Nordin $\mathrm{P}$, Hansson BG, Hansson C, Blohmè I, Larkö O, Andersson K. Human papilloma virus in skin, mouth and uterine cervix in female renal transplant recipients with or without a history of cutaneous squamous cell carcinoma. Acta Derm Venereol. 2007; 87: 219-222. PMID: 17533486

62. Antonsson A, Forslund O, Ekberg H, Sterner G, Hansson BG. The ubiquity and impressive genomic diversity of human skin papillomaviruses suggest a commensalic nature of these viruses. J Virol. 2000; 74: 11636-11641. PMID: 11090162

63. Chouhy D, Bolatti EM, Piccirilli G, Sánchez A, Fernandez Bussy R, Giri AA. Identification of human papillomavirus type 156, the prototype of a new human gammapapillomavirus species, by a generic and highly sensitive PCR strategy for long DNA fragments. J Gen Virol. 2013; 94: 524-533. doi: 10.1099/ vir.0.048157-0 PMID: 23136368

64. Harwood CA, Spink PJ, Surentheran T, Leigh IM, de Villiers EM, McGregor JM, et al. Degenerate and nested PCR: a highly sensitive and specific method for detection of human papillomavirus infection in cutaneous warts. J Clin Microbiol. 1999; 37: 3545-3555. PMID: 10523550

65. Köhler A, Meyer T, Stockfleth E, Nindl I. High viral load of human wart-associated papillomaviruses (PV) but not beta-PV in cutaneous warts independent of immunosuppression. Br J Dermatol. 2009; 161: 528-535. doi: 10.1111/j.1365-2133.2009.09297.x PMID: 19519829

66. Obalek S, Favre M, Szymanczyk J, Misiewicz J, Jablonska S, Orth G. Human papillomavirus (HPV) types specific of epidermodysplasia verruciformis detected in warts induced by HPV3 or HPV3-related types in immunosuppressed patients. J Invest Dermatol. 1992; 98: 936-941. PMID: 1317396

67. Shamanin V, Delius H, de Villiers EM. Development of a broad spectrum PCR assay for papillomaviruses and its application in screening lung cancer biopsies. J Gen Virol. 1994; 75: 1149-1156. PMID: 8176375 
68. Boxman IL, Berkhout RJ, Mulder LH, Wolkers MC, Bouwes Bavinck JN, Vermeer BJ, et al. Detection of human papillomavirus DNA in plucked hairs from renal transplant recipients and healthy volunteers. $J$ Invest Dermatol. 1997; 108: 712-715. PMID: 9129220

69. Boxman IL, Hogewoning A, Mulder LH, Bouwes Bavinck JN, ter Schegget J. Detection of human papillomavirus types 6 and 11 in pubic and perianal hair from patients with genital warts. J Clin Microbiol. 1999; 37: 2270-2273. PMID: 10364596

70. Poljak M, Kocjan BJ, Potočnik M, Seme K. Anogenital hairs are an important reservoir of alpha-papillomaviruses in patients with genital warts. J Infect Dis. 2009; 199: 1270-1274. doi: 10.1086/597619 PMID: 19301978

71. Nobre RJ, Herráez-Hernández E, Fei JW, Langbein L, Kaden S, Gröne HJ, et al. E7 oncoprotein of novel human papillomavirus type 108 lacking the E6 gene induces dysplasia in organotypic keratinocyte cultures. J Virol. 2009; 83: 2907-2916. doi: 10.1128/JVI.02490-08 PMID: 19153227 